\title{
CUBIERTAS VEGETALES EN VIÑEDOS: COMPORTAMIENTO DE MEZCLAS DE LEGUMINOSAS FORRAJERAS ANUALES Y EFECTOS SOBRE LA FERTILIDAD DEL SUELO
}

\author{
Cover crops in vineyards: performance of annual forage legume mixtures and \\ effects on soil fertility
}

\author{
Carlos Ovalle ${ }^{1 *}$, Alejandro del Pozo² ${ }^{\text {Arturo Lavín }}{ }^{3}$, y Juan Hirzel ${ }^{1}$
}

\begin{abstract}
A B S T R A C T
With the objective of evaluating and selecting cover crops to improve soil management in vineyards (Vitis vinifera L.) cv. Cabernet Sauvignon, five cover pastures were evaluated: a) control without vegetation; b) control with spontaneous vegetation; c) legume mixture of early maturing cultivars of subterranean clover (Trifolium subterraneum L.) and burr medic (Medicago polymorpha L.); d) legumes mixture of late maturing cultivars of subterranean clover and balansa clover (T. michelianum Savi); and e) legumes mixture of late maturing cultivars and annual ryegrass (Lolium rigidum Gaud.). The study was conducted at Cauquenes Experimental Center, Agriculture Research Institute (INIA), over two seasons, 2005-2006 and 2006-2007. The experimental design was randomized blocks with four replicates. The three pasture mixtures had a good establishment and achieved high populations. Biomass production was significantly higher $(\mathrm{P} \leq 0.05)$ in the mixtures with late maturing cultivars of subterranean clover and balansa clover, compared to the mixture of early maturing cultivars of subterranean clover and burr medic (2.3 to $4 \mathrm{t} \mathrm{DM} \mathrm{ha}^{-1} \mathrm{yr}^{-1}$ and 5.3 to $6.2 \mathrm{t}$ $\mathrm{DM} \mathrm{ha} \mathrm{Cr}^{-1}$ in the first and second year, respectively). The contents of N, P and $\mathrm{K}$ in the biomass of the cover crops were higher $(P \leq 0.05)$ than those covers with spontaneous species, which increased the content of available $\mathrm{N}$ in the soil. Higher contents of $\mathrm{K}$ and $\mathrm{P}$ were detected in the soil. The cover crops had an important detrimental effect on the population of the spontaneous species that grow in the inter-rows of the vineyard.
\end{abstract}

Key words: Vitis vinifera, Trifolium michelianum, Trifolium subterraneum, Medicago polymorpha, cover crops.

\section{R E S U M E N}

Con el objetivo de evaluar y seleccionar cubiertas vegetales como cultivos entre hileras, para mejorar el manejo del suelo en un viñedo (Vitis vinifera L.) cv. Cabernet Sauvignon, se evaluaron cinco cubiertas: a) testigo sin vegetación; b) testigo con vegetación espontánea; c) mezcla de leguminosas de cultivares precoces de trébol subterráneo (Trifolium subterraneum L.) con hualputra (Medicago polymorpha L.); d) mezcla de cultivares tardíos de trébol subterráneo con trébol balansa (T. michelianum Savi); e) mezcla de leguminosas tardías con ballica anual (Lolium rigidum Gaud.) El ensayo se realizó en el Centro Experimental Cauquenes del Instituto de Investigaciones Agropecuarias (INIA), durante dos temporadas, 2005-2006 y 2006-2007. El diseño experimental fue bloques al azar con cuatro repeticiones. Las tres mezclas forrajeras se establecieron adecuadamente y lograron altas poblaciones. La producción de biomasa fue significativamente más alta $(P \leq 0,05)$ en las mezclas de tréboles tardíos y trébol balansa, respecto de la mezcla de tréboles subterráneos precoces y hualputra $(2,3$ y 4 t MS ha ${ }^{-1}$ año ${ }^{-1}$ y 5,3 y 6,2 t MS ha $^{-1}$ año $^{-1}$ en el primer y segundo año, respectivamente). Los contenidos de N, P y K en la biomasa de las cubiertas fueron mayores que en las cubiertas de especies espontáneas, lo que incrementó el contenido de $\mathrm{N}$ disponible en el suelo. Se encontró un mayor contenido de K y $\mathrm{P}$ en el suelo. Las cubiertas ejercieron un efecto detrimental sobre la población de las especies espontáneas o malezas que crecen en la entre hilera del viñedo.

Palabras clave: Vitis vinifera, Trifolium michelianum, T. subterraneum, Medicago polymorpha, cubiertas vegetales.

\footnotetext{
${ }^{1}$ Instituto de Investigaciones Agropecuarias, Centro Regional de Investigación Quilamapu, Casilla 426, Chillán, Chile.

E-mail: covalle@inia.cl* Autor para correspondencia.

${ }^{2}$ Universidad de Talca, Facultad de Agronomía, Casilla 747, Talca, Chile.

${ }^{3}$ Instituto de Investigaciones Agropecuarias, Centro Experimental Cauquenes, Casilla 165, Cauquenes, Chile.

Recibido: 28 de febrero de $2007 . \quad$ Aceptado: 18 de junio de 2007.
} 


\section{INTRODUCCIÓN}

En la producción silvoagropecuaria destinada al mercado de exportación, como es el caso de la vitivinícola, las exigencias y demandas por la conservación y no contaminación del medio ambiente e inocuidad de los alimentos son en la actualidad indispensables. La implementación de buenas prácticas agrícolas, y el cumplimiento de normas de gestión ambiental que garanticen el manejo sustentable del recurso suelo deben ser implementadas, y la investigación debe aportar con conocimientos sólidos que permitan garantizar que estas prácticas sean compatibles con los objetivos de producción y calidad que la actividad requiere. En el caso particular de la vid, el uso de cubiertas vegetales entre hileras es una tecnología utilizada con este fin en diversas regiones productoras de vinos finos del mundo, como California, Australia, Italia, Francia y otros (Masson y Bertoni, 1996; Nieddu et al., 2000; Masson y Gintzburger, 2000). En Chile su uso es incipiente y no se han investigado los efectos de las cubiertas entre hileras en el sistema suelo-planta de la vid, y en la producción y calidad del vino.

El uso de cubiertas vegetales se ha descrito como una alternativa sustentable de manejo del suelo que presenta múltiples ventajas. Las cubiertas vegetales permiten incrementar el contenido de materia orgánica y de nutrientes en el suelo, debido a la degradación de la biomasa aérea y subterránea; mejorar las propiedades físicas como porosidad, estructura, y estabilidad de los agregados; incrementar la capacidad de retención de humedad y la capacidad de intercambio catiónico del suelo; reducir el escurrimiento del agua y evitar la erosión; e incrementar la actividad biológica en el suelo (Frye y Blevins, 1989). Ventajas adicionales en el caso de frutales y vides son contribuir a la disminución de la población de malezas de difícil control y permitir el control de algunas especies de nemátodos que dañan los huertos frutales (Aballay e Insunza, 2002). Además, diversos trabajos experimentales han demostrado que el manejo inapropiado del suelo afecta las características físico-químicas, lo cual afecta la productividad de la viña y la composición del mosto (Kliewer, 1991; Sicher et al., 1995; Murisier y Zufferey, 1997; Murisier et al., 1999).

La elección de especies y cultivares de plantas para ser usadas como cubiertas entre hileras depende del problema que se pretenda resolver en el viñedo, y de las condiciones edafoclimáticas del sitio. La tendencia actual en viñedos orientados a la producción de vinos de alta calidad, es establecerlos en suelos con pendiente, de perfil poco profundo, de baja retención de humedad y de fertilidad natural baja. En esta situación, el establecimiento de una cubierta vegetal entre las hileras de vides, que contenga leguminosas anuales de autorresiembra, es fundamental para prevenir la erosión hídrica, aportar nitrógeno y otros elementos, sin que ésta compita por agua con el viñedo (Masson y Bertoni, 1996; Masson y Gintzburger, 2000). La elección de las especies que conforman la cubierta vegetal es vital, de manera que el ciclo de crecimiento no interfiera directamente con el crecimiento de la vid y, además, que la cubierta se regenere en los años siguientes a partir de semillas que se producen in situ.

Dentro de las leguminosas forrajeras anuales, capaces de realizar aportes de nitrógeno por fijación, existe una amplia gama de germoplasma proveniente de programas de mejoramiento nacionales y de introducciones de especies y cultivares, que podrían cumplir con este objetivo. Para zonas de precipitaciones limitadas (inferiores a $400 \mathrm{~mm}$ ), la hualputra podría ser una alternativa, ya que presenta una extensa naturalización en Chile (Del Pozo et al., 1989), tiene una amplia variabilidad ecotípica en fenología, capacidad de producción de biomasa y fijación atmosférica de $\mathrm{N}_{2}$ (Del Pozo et al., 2000; 2002; Ovalle et al., 2006b). Para zonas de mayor pluviosidad se dispone de cultivares tardíos de tréboles subterráneos y trébol balansa, los cuales, junto con cultivares de serradela amarilla (Ornithopus compressus L.), serradela rosada (O. sativus Brot.) y biserrula (Biserrula pelecinus L.), debieran ser evaluados. Estas últimas tres especies son especialmente útiles para su establecimiento en suelos pobres de baja fertilidad, pues presentan un adecuado comportamiento en condiciones de bajo contenido de fósforo disponible y acidez edáfica (Ovalle et al., 2006a).

Las especies de leguminosas difieren en su capacidad para fijar $\mathrm{N}$ y en el contenido de $\mathrm{N}$ en la biomasa a nivel de tallos y raíces (Campillo et al., 2003; Sullivan, 2003; Ovalle et al., 2006b), y en consecuencia en la capacidad de aportar $\mathrm{N}$ al viñedo. La transferencia de $\mathrm{N}$ desde la leguminosa ocurre principalmente a través de la descomposición de sus residuos (Danso et al., 1993). Se estima que el $40 \%$ del $\mathrm{N}$ contenido en las plantas de una cu- 
bierta vegetal puede llegar a estar disponible en el suelo el primer año, mientras que el $60 \%$ restante se encontrará disponible si la cubierta vegetal es incorporada como abono verde (Hoyt, 1987). La mineralización del $\mathrm{N}$ aportado por el abono verde $\mathrm{y}$ su utilización por cultivos subsiguientes, depende de la composición química de las leguminosas forrajeras, de las propiedades del suelo, del manejo del abono verde (Harris y Hesterman, 1990; Varco et al., 1993) y de la sincronización entre las leguminosas que liberan $\mathrm{N}$ y la captación por los cultivos subsiguientes que no son leguminosas (Dou et al., 1994; Stute y Posner, 1995).

El uso de gramíneas asociadas a leguminosas también puede ser una alternativa de cubierta vegetal, ya que aumentaría la producción de biomasa y además, presentan sistemas de raíces complementarios al de las leguminosas, lo que tiene un efecto favorable sobre la estructura del suelo (Puchades, 2001).

El objetivo de esta investigación fue estudiar la persistencia y producción de cubiertas vegetales multiespecíficas, evaluar los efectos de las cubiertas en el contenido de materia orgánica y disponibilidad de nutrientes en el suelo, y en la presencia de malezas en la entre hilera del viñedo.

\section{MATERIALES Y MÉTODOS}

El estudio se realizó en el predio El Boldo ( $35^{\circ} 58^{\prime}$ lat. Sur, $72^{\circ} 17^{\prime}$ long. Oeste), comuna de Cauque- nes, Región del Maule, perteneciente al Centro Experimental Cauquenes del Instituto de Investigaciones Agropecuarias (INIA). El suelo es un lomaje granítico, perteneciente a la serie Cauquenes (fine, kaolinitic, mesic Typic Palexeralfs), profundo, de baja fertilidad. El suelo poseía al inicio del ensayo un pH (agua) 6,65; materia orgánica 1,55\%; N 1,93 $\mathrm{mg} \mathrm{kg}^{-1}$; P 5,47 $\mathrm{mg} \mathrm{kg}^{-1}$ y K 207,1 $\mathrm{mg} \mathrm{kg}^{-1}$. El ensayo se estableció sobre una viña var. Cabernet Sauvignon de cinco años de edad, conducida en espaldera vertical.

Los tratamientos evaluados fueron: a) testigo sin cubierta vegetal (T1) mediante la aplicación de glifosato en dosis de $2 \mathrm{~L} \mathrm{ha}^{-1}$ en otoño de cada año); b) testigo con vegetación espontánea (T2); c) mezcla de leguminosas de cultivares precoces de trébol subterráneo con hualputra (T3); d) mezcla de cultivares tardíos de trébol subterráneo con trébol balansa (T4); y e) mezcla de leguminosas tardías con ballica anual (Lolium rigidum Gaud.) (T5). Los cultivares y dosis de semilla de cada tratamiento se indican en el Cuadro 1.

Los tratamientos se establecieron en parcelas de cuatro hileras de $25 \mathrm{~m}$ de largo. La distancia de plantación en la viña era de $2,5 \mathrm{~m}$ entre hilera y $0,5 \mathrm{~m}$ sobre la hilera. El diseño experimental fue de bloques al azar, con cuatro repeticiones.

La preparación del suelo se realizó en mayo de 2005 , realizando una pasada con rastra de discos,

Cuadro 1. Caracterización de los tratamientos, especies, cultivares y dosis de semillas en experimento de cubiertas vegetales en viñedos.

Table 1. Characterization of treatment, species, cultivars, and seed rates on cover crops experiment on vineyards.

\begin{tabular}{|c|c|c|c|c|}
\hline $\mathbf{N}^{\mathbf{o}}$ & Tratamientos & Especie & Cultivar & $\begin{array}{l}\text { Dosis semilla } \\
\quad\left(\mathrm{kg} \mathrm{ha}^{-1}\right)\end{array}$ \\
\hline 1 & Sin cubierta vegetal & & & \\
\hline 2 & Cubierta especies espontáneas & & & \\
\hline \multirow[t]{2}{*}{3} & \multirow[t]{2}{*}{ Cubierta de leguminosas precoces } & Trébol subterráneo & Seaton Park & 12 \\
\hline & & Hualputra & Santiago & 8 \\
\hline \multirow[t]{2}{*}{4} & \multirow[t]{2}{*}{ Cubierta de leguminosa tardías } & Trébol subterráneo & Antas & 12 \\
\hline & & Trébol balansa & Paradana & 5 \\
\hline \multirow[t]{3}{*}{5} & \multirow{3}{*}{$\begin{array}{l}\text { Cubierta de leguminosa tardías } \\
+ \text { gramínea }\end{array}$} & Trébol subterráneo & Antas & 12 \\
\hline & & Trébol balansa & Paradana & 5 \\
\hline & & Ballica & Wimmera & 10 \\
\hline
\end{tabular}


luego dos pasadas de arado cincel, emparejando el suelo con un rastrillo manual. La siembra se realizó con una máquina sembradora manual (Planet Jr. 300A, Allen \& Co., Philadelphia, Pennsylvania, USA), el 31 de mayo de 2005, en hileras separadas a $20 \mathrm{~cm}$ y dispuestas en el mismo sentido de la plantación. Las semillas de leguminosas se inocularon con el rizobio específico en dosis de $10 \mathrm{~g}$ de inóculo $\mathrm{kg}^{-1}$ de semilla.

La fertilización para el establecimiento fue la aplicación al voleo y posterior incorporación de $150 \mathrm{~kg} \mathrm{ha}^{-1}$ de $\mathrm{P}_{2} \mathrm{O}_{5}$ como superfosfato triple, 500 $\mathrm{kg} \mathrm{ha}^{-1}$ de $\mathrm{CaSO}_{4}$ como fertiyeso, $85 \mathrm{~kg} \mathrm{ha}^{-1}$ de $\mathrm{K}_{2} \mathrm{O}$ como cloruro de potasio, $200 \mathrm{~kg} \mathrm{ha}^{-1}$ de $\mathrm{S}$ como sulpomag, y $20 \mathrm{~kg} \mathrm{ha}^{-1}$ de $\mathrm{B}$ como boronatrocalcita. Las parcelas recibieron anualmente una fertilización de mantención de $50 \mathrm{~kg} \mathrm{ha}^{-1}$ de $\mathrm{P}_{2} \mathrm{O}_{5}$ como superfosfato triple. En ambos años la viña se regó por goteo entre los meses de noviembre y marzo.

\section{Evaluaciones en la cubierta vegetal}

La densidad de plantas se evaluó al segundo mes de inicio de la temporada de crecimiento, mediante el recuento de plántulas a partir de muestras obtenidas de un cilindro de bronce de $78,5 \mathrm{~cm}^{2}$, el cual se lanzó al azar cinco veces por cada tratamiento y repetición. Luego en laboratorio, en cada muestra se contabilizaron las especies en el área del cilindro.

La producción de fitomasa se evaluó mediante el corte circunscrito en cuadrantes de $1 \mathrm{~m}^{2}$, a razón de cinco muestras por parcela. El corte de la fitomasa se realizó a $5 \mathrm{~cm}$ del suelo abarcando la totalidad de la biomasa aérea contenida en el cuadrante. En el primer año se efectuó un muestreo el 31 de octubre de 2005. En el segundo año se realizaron tres muestreos (15 de agosto, 21 de septiembre y 3 de noviembre de 2006), y con éstos se determinó la disponibilidad instantánea total de forraje, sin excluir las especies espontáneas de las sembradas. Las muestras se secaron en horno con ventilación forzada a $70{ }^{\circ} \mathrm{C}$ por $72 \mathrm{~h}$.

La composición botánica y cobertura de la vegetación se evaluó en el período de máxima expresión de la vegetación herbácea (primavera) utilizando el método "point quadrat" (Daget y Poissonet, 1971). En las diagonales de las parcelas elegidas al azar, se dispusieron líneas de $4 \mathrm{~m}$ de lar- go, midiéndose 100 puntos cada $4 \mathrm{~cm}$; la proporción de cada especie se expresó a través de la contribución específica de contacto (CSC, \%).

Se determinó el contenido de N, P y K de la biomasa de la cubierta entre hilera, con el objeto de cuantificar los aportes y/o extracción de cada tipo de cubierta. De acuerdo a la metodología propuesta por Sadzawka et al. (2004) se analizó: materia seca, obtenida a través de secado en estufa a 65 ${ }^{\circ} \mathrm{C}$ durante $48 \mathrm{~h}$; contenido de nitrógeno mediante digestión Kjeldahl y determinación por destilación y titulación automática; contenido de $\mathrm{P}$ y de $\mathrm{K}$ por digestión ácida con $\mathrm{HCl} 2 \mathrm{M}$ de las cenizas obtenidas por calcinación de la muestra a $500{ }^{\circ} \mathrm{C}$; determinación por colorimetría del fosfo-vanadomolíbdico en el caso del $\mathrm{P}$, y por espectrofotometría de llama por emisión atómica para el K.

\section{Evaluaciones en el suelo}

Para conocer la evolución de las variables químicas del suelo: materia orgánica, $\mathrm{pH}, \mathrm{N}, \mathrm{P}$ y K, se realizó un seguimiento en el tiempo en tres tratamientos: sin cubierta vegetal, cubierta de leguminosas precoces y cubierta de leguminosas tardías. Para este efecto, se usó una modificación del método de incubación de suelos in situ propuesto por Raison et al. (1987). Al inicio del experimento se instalaron tubos de PVC (polivinil cloruro) en el suelo, cuyas dimensiones fueron $25 \mathrm{~cm}$ de largo y $10 \mathrm{~cm}$ de diámetro. El número de tubos por unidad experimental fue equivalente al número de muestreos a realizar durante la temporada, en total cuatro tubos. En ellos se dispuso la biomasa aérea proveniente de cada corte de la cubierta vegetal. Para ello, se calculó la disponibilidad de materia seca de la pradera, y se dispuso en cada tubo la cantidad de fitomasa proporcional al área del tubo $\left(74,6 \mathrm{~cm}^{2}\right)$. Periódicamente, cada 30 a 45 días, se colectó un tubo por unidad experimental, y las muestras de suelo correspondientes se sometieron a análisis de materia orgánica (MO) por el método Walkey-Black modificado, oxidación con $\mathrm{K}_{2} \mathrm{Cr}_{2} \mathrm{O}_{7}$ y determinación por colorimetría; $\mathrm{N}$ mineral por $\mathrm{KCl} 2 \mathrm{~N} \mathrm{1:10}$ y lectura en un autoanalizador a inyección de flujo segmentado (SKALAR, SA 4000, Breda, The Netherlands); P extractable Olsen por bicarbonato de sodio $0,5 \mathrm{M}$ a $\mathrm{pH} 8,5 \mathrm{y}$ determinación por colorimetría; y $\mathrm{K}$ intercambiable por extracción con solución de acetato de amonio $1 \mathrm{~mol} \mathrm{~L}^{-1}$, pH 7,0 y determinación por espectrofotometría de absorción y emisión atómica. 


\section{RESULTADOS Y DISCUSIÓN}

\section{Establecimiento y autorresiembra de las cubier- tas vegetales}

El establecimiento de las cubiertas vegetales fue óptimo en los diferentes tratamientos, logrando una implantación de las especies sembradas en el primer año de entre 600 y 800 plantas $\mathrm{m}^{-2}$ (Cuadro 2). Esta población fue superior a los estándares de medición de la calidad del establecimiento de una pradera de leguminosas anuales, que sitúan la población en aproximadamente 500 plantas $\mathrm{m}^{-2}$ para un buen establecimiento (Puckridge y French, 1983). La población de tréboles subterráneos fue significativamente mayor $(\mathrm{P} \leq 0,05)$ en las mezclas que no incluyeron gramínea. Destaca la población de plantas significativamente $(\mathrm{P} \leq 0,05)$ alta del tratamiento de vegetación espontánea, que alcanzó 2.363 plantas $\mathrm{m}^{-2}$ (Cuadro 2). En la resiembra natural del segundo año, en general, se obtuvieron altas poblaciones de las especies sembradas. La densidad poblacional del trébol subterráneo fue particularmente elevada en la mezcla que incluye el cultivar precoz Seaton Park, debido por una parte a la menor dureza seminal de este cultivar (Ovalle et al., 2003), y por la mayor asignación de recursos para la reproducción (producción de semillas) que realizan las leguminosas precoces y en particular la hualputra (Del Pozo et al., 2002). Esta mayor producción de semillas debiera estar asociada a una mayor persistencia de la cubierta, lo que deberá corroborarse en los años sucesivos de este estudio (Del Pozo et al., 2002).
La densidad poblacional del trébol balansa y de la hualputra fue baja en el año 2, debido a la alta dureza seminal de dichas especies (Ovalle et al., 1997a; 1997b). Al igual que en el primer año, destaca la población significativamente $(\mathrm{P} \leq 0,05)$ alta del tratamiento de vegetación espontánea, que alcanzó en este segundo año a 7.317 plantas $\mathrm{m}^{-2}$ (Cuadro 2).

\section{Producción de fitomasa}

Las mezclas de leguminosas forrajeras anuales se establecieron y produjeron de manera óptima en el primer y segundo año. La producción de fitomasa de las cubiertas de leguminosas tardías con o sin gramíneas fue significativamente más alta $(\mathrm{P} \leq 0,05)$ en el segundo año, que la producción de la cubierta de cultivares precoces y de la vegetación espontánea. En ambos años la producción de las cubiertas sembradas fue alta, pero en particular en la segunda temporada cuando las producciones superaron los $5.300 \mathrm{~kg} \mathrm{MS} \mathrm{ha}^{-1}$ año $^{-1}$ (Cuadro 3). Los resultados son comparables, y en algunos casos superiores, a los mostrados por las mismas especies bajo condiciones de praderas permanentes de pastoreo en la misma área agroecológica (Avendaño et al., 2005; Ovalle et al., 2005).

\section{Composición botánica}

En los dos años de evaluación el trébol subterráneo mostró la mayor contribución específica de contacto (CSC), respecto de las otras especies constituyentes de las mezclas (Cuadro 4). En la primera temporada la CSC del trébol subterráneo fue significativamente menor $(\mathrm{P} \leq 0,05)$ en el tratamiento en

Cuadro 2. Densidad de plantas $\left(\mathrm{pl} \mathrm{m}^{-2}\right)$ de diferentes cubiertas vegetales sembradas entre las hileras del viñedo, en el año de establecimiento (2005-2006) y en el segundo año (2006-2007).

Table 2. Plant density $\left(\mathrm{pl} \mathrm{m}^{-2}\right)$ of different cover crops sown in the inter rows in vineyard in the first (2005-2006) and second years (2006-2007).

\begin{tabular}{|c|c|c|c|c|c|c|}
\hline Tratamientos & $\begin{array}{c}\text { Trébol } \\
\text { subterráneo }\end{array}$ & $\begin{array}{c}\text { Trébol } \\
\text { balansa }\end{array}$ & Hualputra & $\begin{array}{c}\text { Ballica } \\
\text { Wimmera }\end{array}$ & Otras & Total \\
\hline \multicolumn{7}{|l|}{2005} \\
\hline Vegetación espontánea & & & & & $2.363 \mathrm{a}$ & $2.363 \mathrm{a}$ \\
\hline Leguminosas precoces & $519 \mathrm{a}$ & & 281 & & $781 \mathrm{~b}$ & $1.581 \mathrm{~b}$ \\
\hline Leguminosas tardías & $513 \mathrm{a}$ & $163 \mathrm{a}$ & & & $613 b$ & $1.288 \mathrm{~b}$ \\
\hline Leguminosas tardías + ballica & $331 \mathrm{~b}$ & $219 \mathrm{a}$ & & 156 & $763 \mathrm{~b}$ & $1.469 \mathrm{~b}$ \\
\hline \multicolumn{7}{|l|}{2006} \\
\hline Vegetación espontánea & - & - & - & - & $7.317 \mathrm{a}$ & $7.317 \mathrm{a}$ \\
\hline Leguminosas precoces & $3.669 \mathrm{a}$ & - & 63 & - & $931 \mathrm{~b}$ & $4.663 \mathrm{~b}$ \\
\hline Leguminosas tardías & $2.256 \mathrm{~b}$ & $106 \mathrm{a}$ & - & - & $875 \mathrm{~b}$ & $3.238 \mathrm{c}$ \\
\hline Leguminosas tardías + ballica & $1.600 \mathrm{~b}$ & $188 \mathrm{a}$ & - & 100 & $1.275 \mathrm{~b}$ & $3.163 \mathrm{c}$ \\
\hline
\end{tabular}

Medias seguidas por letras distintas en una misma columna difieren significativamente según prueba de Duncan $(\mathrm{P} \leq 0,05)$. 
Cuadro 3. Fitomasa total y de las especies sembradas de cubiertas vegetales en viñedos establecidos en un suelo granítico, en dos estaciones de crecimiento.

Table 3. Total and sown biomass of species of cover crops in a vineyard in a granitic soil, in two growing seasons.

\begin{tabular}{|c|c|c|c|c|}
\hline \multirow[t]{2}{*}{ Tratamientos } & \multicolumn{2}{|c|}{ 2005-2006 } & \multicolumn{2}{|c|}{ 2006-2007 } \\
\hline & $\begin{array}{c}\text { Total } \\
\left(\mathrm{kg} \mathrm{ha}^{-1}\right)\end{array}$ & $\begin{array}{c}\text { Especie sembrada } \\
\left(\mathrm{kg} \mathrm{ha}^{-1}\right)\end{array}$ & $\begin{array}{c}\text { Total } \\
\left(\mathrm{kg} \mathrm{ha}^{-1}\right)\end{array}$ & $\begin{array}{c}\text { Especie sembrada } \\
\left(\mathrm{kg} \mathrm{ha} \mathbf{a}^{-1}\right)\end{array}$ \\
\hline Vegetación espontánea & $410 \mathrm{c}$ & - & $320 \mathrm{c}$ & - \\
\hline Leguminosas precoces & $3.460 \mathrm{~b}$ & $2.330 \mathrm{~b}$ & $5.445 \mathrm{~b}$ & $5.391 \mathrm{~b}$ \\
\hline Leguminosas tardías & $4.160 \mathrm{a}$ & $4.070 \mathrm{a}$ & $6.148 \mathrm{a}$ & $6.148 \mathrm{a}$ \\
\hline Leguminosas tardías + ballica & $4.010 \mathrm{a}$ & $3.900 \mathrm{a}$ & $6.623 \mathrm{a}$ & $6.623 \mathrm{a}$ \\
\hline
\end{tabular}

Medias seguidas por letras distintas en una misma columna difieren significativamente según prueba de Duncan $(P \leq 0,05)$. -: no corresponde.

que se incluyeron cultivares tardíos asociados con ballica anual. En la segunda temporada no se observaron diferencias en la CSC del trébol subterráneo entre los tratamientos. En ambas temporadas la contribución del trébol balansa fue baja, no presentando diferencia significativa $(\mathrm{P} \geq 0,05)$ con el tratamiento de leguminosas tardías y ballica. En el caso de la hualputra y la ballica, ninguna de ellas tuvo un aporte importante, presentando una contribución no superior al 2 y $5 \%$, respectivamente (Cuadro 4).

Las principales especies espontáneas o malezas presentes en el ensayo fueron Rumex acetosella L., Hypochaeris glabra L., Plantago lanceolata L., Hypochaeris radicata L., Leontodon saxatilis Lam., Avena barbata Pott ex Link, y Lolium multiflorum Lam. Respecto del efecto sobre el control de otras especies de malezas que se desarrollan en la entrehilera del viñedo, la participación de las especies sembradas en la cubierta fue superior al $95 \%$ en todos los tratamientos (Cuadro 4), con lo cual se realiza un adecuado control de las malezas en la entre hilera de la viña.

\section{Composición química de la biomasa de las cu- biertas}

El contenido de $\mathrm{N}$ de las mezclas de leguminosas fue alto y no mostró diferencias significativas $(\mathrm{P} \geq$ 0,05 ) entre las diferentes cubiertas evaluadas (Cuadro 5). La alta producción de biomasa, que en ambos años fue mayor en la mezcla de leguminosas tardías, y el alto contenido de $\mathrm{N}$ de esta biomasa, que osciló entre 2,91 y $3,15 \%$, permiten suponer un aporte e ingreso de $\mathrm{N}$ altos al sistema. Parte de este $\mathrm{N}$ proviene del stock del suelo, pero la fracción más importante proviene de la fijación bioló-

Cuadro 4. Composición botánica (\%) de las cubiertas vegetales en viñedos establecidos en suelos graníticos, en dos temporadas de crecimiento.

Table 4. Botanical composition (\%) of cover crops in vineyard established in granitic soils, in two growing seasons.

\begin{tabular}{|c|c|c|c|c|c|}
\hline Tratamiento & $\begin{array}{c}\text { Trébol } \\
\text { subterráneo }\end{array}$ & $\begin{array}{c}\text { Trébol } \\
\text { balansa }\end{array}$ & Hualputra & $\begin{array}{c}\text { Ballica } \\
\text { Wimmera }\end{array}$ & Otras \\
\hline \multicolumn{6}{|l|}{$2005-2006$} \\
\hline Vegetación espontánea & - & - & - & - & $100 \mathrm{a}$ \\
\hline Leguminosas precoces & $92 \mathrm{a}$ & - & 2 & - & $6 \mathrm{~b}$ \\
\hline Leguminosas tardías & $88 \mathrm{ab}$ & $10 \mathrm{a}$ & - & - & $2 \mathrm{~b}$ \\
\hline Leguminosas tardías + ballica & $77 \mathrm{~b}$ & $15 \mathrm{a}$ & - & 5 & $3 \mathrm{~b}$ \\
\hline \multicolumn{6}{|l|}{$2006-2007$} \\
\hline Vegetación espontánea & - & - & - & - & $100 \mathrm{a}$ \\
\hline Leguminosas precoces & $96 \mathrm{a}$ & - & 3 & - & $1 \mathrm{~b}$ \\
\hline Leguminosas tardías & $95 \mathrm{a}$ & $5 \mathrm{a}$ & - & - & $0 \mathrm{~b}$ \\
\hline Leguminosas tardías + ballica & $93 \mathrm{a}$ & $4 \mathrm{a}$ & - & 3 & $0 \mathrm{~b}$ \\
\hline
\end{tabular}

Medias seguidas por letras distintas en una misma columna difieren significativamente según prueba de Duncan (P $\leq 0,05)$. -: no corresponde. 
gica. En efecto, en suelos graníticos del secano interior la fijación en trébol subterráneo ha sido estimada en más del $90 \%$ del $\mathrm{N}$ contenido en la biomasa, lo que equivale a niveles de fijación de aproximadamente 16 a $18 \mathrm{~kg} \mathrm{~N}_{\text {ha }}{ }^{-1} \mathrm{año}^{-1}$ por cada tonelada de materia seca producida (Ovalle et al., 2006b). En $\mathrm{P}$ no se observaron diferencias significativas $(\mathrm{P}$ $\geq 0,05)$ en los distintos tratamientos, presentando contenidos que variaron entre un 0,33 y $0,39 \%$. En el caso del K, los tratamientos de leguminosas tar- días solas o con ballica presentaron los valores más altos para $\mathrm{K}$, siendo estadísticamente superiores ( $\mathrm{P}$ $\leq 0,05)$ al resto de los tratamientos, con valores similares entre sí $(3,61$ y $3,62 \%)$ (Cuadro 5).

\section{Evolución del contenido de nutrientes del suelo} El pH y el contenido de $\mathrm{MO}$ en los primeros $20 \mathrm{~cm}$ del suelo no se vieron modificados por la inclusión de cubiertas vegetales entre las hileras del viñedo después de 2 años de realizada la siembra (Cuadro 6).

Cuadro 5. Composición química de las cubiertas vegetales. Octubre 2005.

Table 5. Chemical composition of cover crops. October 2005.

\begin{tabular}{lccc}
\hline Tratamientos & $\mathbf{N}$ & $\mathbf{P}$ & $\mathbf{K}$ \\
\cline { 2 - 4 } & \multicolumn{3}{c}{} \\
\hline Vegetación espontánea & $2,86 \mathrm{a}$ & $0,39 \mathrm{a}$ & $2,89 \mathrm{~b}$ \\
Leguminosas precoces & $2,91 \mathrm{a}$ & $0,35 \mathrm{a}$ & $3,02 \mathrm{~b}$ \\
Leguminosas tardías & $3,15 \mathrm{a}$ & $0,35 \mathrm{a}$ & $3,62 \mathrm{a}$ \\
Leguminosas tardías + ballica & $2,96 \mathrm{a}$ & $0,33 \mathrm{a}$ & $3,61 \mathrm{a}$ \\
\hline
\end{tabular}

Medias seguidas por letras distintas en una misma columna difieren significativamente según prueba de Duncan $(\mathrm{P} \leq 0,05)$

Cuadro 6. Variación en el tiempo del pH, del contenido de materia orgánica (MO), N, P y K en el suelo, bajo diferentes cubiertas vegetales en viñedos establecidos en suelos graníticos, durante la temporada 2006-2007.

Table 6. Time variation of $\mathrm{pH}$, soil content of organic matter (MO), N, P, and $\mathrm{K}$ under different cover crops in vineyards in granitic soils, during the 2006-2007 growing season.

\begin{tabular}{|c|c|c|c|c|}
\hline \multirow[t]{2}{*}{ Tratamiento } & \multicolumn{4}{|c|}{ Fechas de muestreo } \\
\hline & 11/08/06 & 09/09/06 & 23/09/06 & 09/11/06 \\
\hline \multicolumn{5}{|l|}{$\mathrm{pH}$} \\
\hline Control sin cubierta vegetal & $6,65 \mathrm{a}$ & $6,70 \mathrm{a}$ & $6,43 \mathrm{a}$ & $6,42 \mathrm{a}$ \\
\hline Cubierta de leguminosas precoces & $6,48 \mathrm{a}$ & $6,28 \mathrm{a}$ & $6,73 \mathrm{a}$ & $6,66 \mathrm{a}$ \\
\hline Cubierta de leguminosas tardías & $6,77 \mathrm{a}$ & $6,81 \mathrm{a}$ & $6,53 \mathrm{a}$ & $6,50 \mathrm{a}$ \\
\hline \multicolumn{5}{|l|}{$\mathrm{MO}, \%$} \\
\hline Control sin cubierta vegetal & $1,55 \mathrm{a}$ & $1,61 \mathrm{~b}$ & $1,68 \mathrm{a}$ & $1,55 \mathrm{a}$ \\
\hline Cubierta de leguminosas precoces & $1,86 \mathrm{a}$ & $1,90 \mathrm{ab}$ & $2,17 \mathrm{a}$ & $2,07 \mathrm{a}$ \\
\hline Cubierta de leguminosas tardías & $1,71 \mathrm{a}$ & $2,44 \mathrm{a}$ & $2,22 \mathrm{a}$ & $2,12 \mathrm{a}$ \\
\hline \multicolumn{5}{|l|}{$\mathrm{N}, \mathrm{mg} \mathrm{kg}^{-1}$} \\
\hline Control sin cubierta vegetal & $1,9 \mathrm{~b}$ & $6,8 \mathrm{c}$ & $9,5 \mathrm{~b}$ & $5,1 \mathrm{c}$ \\
\hline Cubierta de leguminosas precoces & $21,9 \mathrm{a}$ & $62,6 \mathrm{~b}$ & $26,9 \mathrm{a}$ & $17,0 \mathrm{~b}$ \\
\hline Cubierta de leguminosas tardías & $14,1 \mathrm{a}$ & $193,7 \mathrm{a}$ & $39,8 \mathrm{a}$ & $44,7 \mathrm{a}$ \\
\hline \multicolumn{5}{|l|}{$\mathrm{P}_{2} \mathrm{O}_{5}, \mathrm{mg} \mathrm{kg}^{-1}$} \\
\hline Control sin cubierta vegetal & $5,5 \mathrm{~b}$ & $7,5 \mathrm{~b}$ & $6,4 \mathrm{~b}$ & $5,2 \mathrm{~b}$ \\
\hline Cubierta de leguminosas precoces & $12,9 \mathrm{a}$ & $19,4 \mathrm{a}$ & $16,1 \mathrm{a}$ & $18,2 \mathrm{a}$ \\
\hline Cubierta de leguminosas tardías & $15,5 \mathrm{a}$ & $23,1 \mathrm{a}$ & $18,6 \mathrm{a}$ & $18,7 \mathrm{a}$ \\
\hline \multicolumn{5}{|l|}{$\mathrm{K}_{2} \mathrm{O}, \mathrm{mg} \mathrm{kg}^{-1}$} \\
\hline Control sin cubierta vegetal & $208 \mathrm{~b}$ & $252 \mathrm{~b}$ & $226 \mathrm{~b}$ & $214 b$ \\
\hline Cubierta de leguminosas precoces & $279 \mathrm{a}$ & $438 \mathrm{a}$ & $474 \mathrm{a}$ & $442 \mathrm{a}$ \\
\hline Cubierta de leguminosas tardías & $287 \mathrm{a}$ & $557 \mathrm{a}$ & $521 \mathrm{a}$ & $505 \mathrm{a}$ \\
\hline
\end{tabular}


El contenido de $\mathrm{N}$ mineral presentó diferencias significativas $(\mathrm{P} \leq 0,05)$ entre tratamientos en el período entre julio y noviembre. Este comportamiento se explica por la activa mineralización favorecida por las adecuadas condiciones ambientales del suelo. Una situación similar fue observada en cubiertas vegetales en huertos orgánicos de frambuesa (Rubus idaeus L.) en Chile, en estudios realizados en suelos trumaos de riego del valle central (Ovalle et al., 2007a; 2007b). El contenido de $\mathrm{P}$ del suelo fue significativamente más alto en los tratamientos con cubierta que recibieron fertilización de establecimiento y mantención (equivalente a 150 y $50 \mathrm{~kg} \mathrm{P}_{2} \mathrm{O}_{5}$ ha $^{-1}$, respectivamente). Respecto del K, se observó también un significativo aumento en su contenido producto de la fertilización de establecimiento y de la mineralización de la MO.

Los efectos descritos anteriormente serán analizados desde el punto de vista del comportamiento productivo y del crecimiento de la vid en otro artículo.

\section{CONCLUSIONES}

La incorporación de cubiertas vegetales entre las hileras de un viñedo Cabernet Sauvignon en suelos graníticos del secano interior mediterráneo subhúmedo de Chile, presentó ventajas en términos de manejo del suelo, al ser comparado con un suelo manejado libre de vegetación o con la vegetación espontánea. Las tres mezclas forrajeras evaluadas experimentaron un adecuado comportamiento en términos de establecimiento y autosiembra al segundo año; las cubiertas vegetales compuestas por cultivares tardíos de trébol subterráneo y trébol balansa con o sin ballica anual, produjeron más biomasa en ambos años. La incorporación de $\mathrm{N}$ por fijación biológica de las leguminosas y su posterior mineralización en el suelo, permitió incrementar el contenido de $\mathrm{N}$ disponible en éste. Por el mismo efecto, y debido a la fertilización de establecimiento que recibió el suelo bajo cubiertas vegetales, también se encontró un mayor tenor de $\mathrm{K}$ en el suelo. El contenido de $\mathrm{P}$ se vio asimismo incrementado principalmente por los aportes de fertilización fosfatada necesarios para el establecimiento y mantención de las cubiertas. Por último, se determinó que las cubiertas ejercieron un efecto detrimental sobre la población de las especies espontáneas o malezas que crecen en la entre hilera del viñedo.

\section{LITERATURA CITADA}

Aballay, E., y V. Insunza. 2002. Evaluación de plantas con propiedades nematicidas en el control de Xiphinema index en uva de mesa cv. Thompson Seedless en la zona central de Chile. Agric. Téc. (Chile) 62:357365.

Avendaño, J., C. Ovalle, A. Del Pozo, F. Fernández, y C. Porqueddu. 2005. Mezclas de trébol subterráneo con otras leguminosas anuales para suelos graníticos del secano mediterráneo subhúmedo de Chile. Agric. Téc. (Chile) 65:165-176.

Campillo, R., S. Urquiaga, I. Pino, y A. Montenegro. 2003. Estimación de la fijación biológica de nitrógeno en leguminosas forrajeras mediante la metodología del 15N. Agric. Téc. (Chile) 63:169-179.

Daget, Ph., et J. Poissonet. 1971. Une méthode d'analyse phytologique des prairies, critères dфcapplication. Ann. Agron. 22:5-41.
Danso, S., F. Palmason, and G. Hardarson. 1993. Is nitrogen transferred between field crops? Examining the question through a sweet-blue lupin (Lupinus angustifolius L.)-oats (Avena sativa) intercrop. Soil Biol. Biochem. 25:1135-1137.

Del Pozo, A., C. Ovalle, J. Aronson, and J. Avendaño. 2000. Developmental responses to temperature and photoperiod in ecotypes of Medicago polymorpha $\mathrm{L}$. collected along an environmental gradient in central Chile. Ann. Bot. (London) 85:809-814.

Del Pozo, A., C. Ovalle, J. Aronson, and J. Avendaño. 2002. Ecotypic differentiation in Medicago polymorpha along an environmental gradient in central Chile. II. Winter growth as related to phenology and temperature. Plant Ecol. 160:53-59.

Del Pozo, A., C. Ovalle, y J. Avendaño. 1989. Los medicagos anuales. I. Comparación con Australia. Agric. Téc. (Chile) 49:260-267. 
Dou, Z., R.H. Fox, and J.D. Toth. 1994. Tillage effect on seasonal nitrogen availability in corn supplied with legume green manures. Plant Soil 162:203-210.

Frye, W.W., and R.L. Blevins. 1989. Economically sustainable crop production with legume cover crops and conservation tillage. J. Soil Water Conserv. 44:57-60.

Harris, G.H., and O.B. Hesterman. 1990. Quantifying the nitrogen contribution from alfalfa to soil and two succeeding crops using nitrogen-15. Agron. J. 82:129-134.

Hoyt, G.D. 1987. Legumes as a green manure in conservation tillage. p. 96-98. In J.F. Powers (ed.) The role of legumes in conservation tillage systems. Soil Conservation Society of America, Ankeny, Iowa, USA.

Kliewer, W.M. 1991. Methods for determining the nitrogenous status of vineyards. p. 133-147. Proc. Int. Symp. on Nitrogen in Grapes and Wines, Seattle. 1819 June. American Society for Enology and Viticulture (ASEV), Seattle, Washington, USA.

Masson, P., et G. Bertoni. 1996. Essai d'enherbement d'un vignoble méridional á base de trèfle souterrain: synthèse de six années d'expérimentation. p. 140-142. In Actes de $11^{\mathrm{e}}$ Colloque Begrünung im Weinbau. 2831 août. Internationaler Arbeitskreis im Weinbau, Kaltern, Sud Tyrol, Italie.

Masson, P., and G. Gintzburger. 2000. Les légumineuses fourragères dans les systèmes de production méditerranéens: utilisations alternatives. Cahiers Options Méditerranéennes 45:395-406.

Murisier, F., D. Maigre, e J.L. Spring. 1999. Gestione del suolo nella viticoltura svizzera. Esperienze con varie tecniche di inerbimento e riflessi sulla qualità del vino. Notiziario Tecnico CRPV 58:1-17.

Murisier, F., et V. Zufferey. 1997. Rapport feuille-fruit de la vigne et qualité du raisin. Revue Suisse Vitic. Arboric. Hortic. 29:355-362.

Nieddu, G., O. Graviano, A. Lostia, and C. Porqueddu. 2000. Effects of Medicago polymorpha cover cropping in Sardinia vineyards. Cahiers Options Méditerranéennes 45:449-452.

Ovalle, C., S. Arredondo, y O. Romero. 2006a. Serradela amarilla (Ornithopus compressus) y serradela rosada (O. sativus): dos nuevas especies de leguminosas forrajeras anuales para la zona mediterránea de Chile. Agric. Téc. (Chile) 66:196-209.

Ovalle, C., A. Del Pozo, J. Avendaño, y J. Aronson. 1997a. Características fenológicas y productivas de 34 accesiones de hualputra (Medicago polymorpha) colectadas en la zona mediterránea de Chile. Agric. Téc. (Chile) 57:261-270.

Ovalle, C., A. Del Pozo, J. Avendaño, P. Bustos, y S. Arredondo. 2003. Caracterización preliminar de una colección de leguminosas forrajeras anuales para la zona mediterránea de Chile. Agric. Téc. (Chile) 63:156-168.
Ovalle, C., A. Del Pozo, J. Avendaño, y F. Fernández. 2005. Adaptación, crecimiento y producción de nuevas leguminosas forrajeras anuales en la zona mediterránea de Chile. II. Comportamiento de las especies en suelos graníticos del secano interior subhúmedo. Agric. Téc. (Chile) 65:265-277.

Ovalle, C., M.I. González, J. Hirzel, I. Pino, A. Del Pozo, and S. Urquiaga. 2007a. Contribution and transfer of nitrogen from cover crops to raspberry plant using isotopic techniques with ${ }^{15} \mathrm{~N}$. Acta Hortic. En prensa.

Ovalle, C., M.I. González, A. Del Pozo, J. Hirzel, y V. Hernaíz. 2007b. Cubiertas vegetales en producción orgánica de frambuesa: efectos sobre el contenido de nutrientes del suelo y en el crecimiento y producción de las plantas. Agric. Téc. (Chile) 67:271-280.

Ovalle, C., F. Squella, S. Arredondo, A. Del Pozo, y J. Avendaño. 1997b. Trébol balansa (Trifolium michelianum) una leguminosa forrajera promisoria para sistemas pastorales del secano mediterráneo de Chile. Agric. Téc. (Chile) 57:50-57.

Ovalle, C., S. Urquiaga, A. Del Pozo, E. Zagal, y S. Arredondo. 2006b. Nitrogen fixation in six forage legumes in mediterranean central Chile. Acta Agric. Scand. 56:277-293.

Puchades, J. 2001. Empleo de cubiertas vegetales en cítricos. Valencia, España. Disponible en http:// www.docum.com/huerta/cubiertasencitricos.htm Leído el 9 de diciembre 2004.

Puckridge, D.W., and R.J. French. 1983. The annual legume pasture in cereal-ley farming systems of Southern Australia. A review. Agric. Ecosyst. Environ. 9:229-267.

Raison, R.J., M.J. Connell, and P.K. Khanna. 1987. Methodology for studying fluxes of soil mineral $\mathrm{N}$ in situ. Soil Biol. Biochem. 19:521-530.

Sicher, L., A. Dorigoni, and G. Stringari. 1995. Soil management effects on nutritional status and grapevine performances. Second International Symposium on Diagnosis of Nutritional Status of Deciduos Fruit Orchards. Acta Hortic. 383:73-82.

Stute, J.K., and J.L. Posner. 1995. Synchrony between legume nitrogen release and corn demand in the upper Midwest. Agron. J. 87:1063-1069.

Sadzawka R., A., R. Grez Z., M.A. Carrasco R., y M.L. Mora G. 2004. Métodos de análisis de tejidos vegetales. 53 p. Sociedad Chilena de la Ciencia del Suelo, Comisión de Normalización y Acreditación, Santiago, Chile.

Sullivan, P. 2003. Overview of cover crops and green manures. 15 p. Technical Publication. ATTRA. National Sustainable Agriculture Information Service, Fayetteville, Arkansas, USA.

Varco, J., M. Frye, S. Smith, and J. MacKown. 1993. Tillage effects on legume decomposition and transformation of legume and fertilizer nitrogen-15. Soil Sci. Soc. Am. J. 57:750-756 